\title{
Texture features based microscopic image classification of liver cellular granuloma using artificial neural networks
}

Conference or Workshop Item

Accepted Version

Shi, F., Chen, G., Wang, Y., Yang, N., Chen, Y., Dey, N. and Sherratt, R. S. (2019) Texture features based microscopic image classification of liver cellular granuloma using artificial neural networks. In: IEEE 8th Joint International Information Technology and Artificial Intelligence Conference (ITAIC), 2426 May 2019, Chongqing, China, pp. 432-439. doi:

https://doi.org/10.1109/ITAIC.2019.8785563 Available at http://centaur.reading.ac.uk/90511/

It is advisable to refer to the publisher's version if you intend to cite from the work. See Guidance on citing.

Published version at: http://dx.doi.org/10.1109/ITAIC.2019.8785563

To link to this article DOI: http://dx.doi.org/10.1109/ITAIC.2019.8785563 
including copyright law. Copyright and IPR is retained by the creators or other copyright holders. Terms and conditions for use of this material are defined in the End User Agreement.

\section{www.reading.ac.uk/centaur}

\section{CentAUR}

Central Archive at the University of Reading

Reading's research outputs online 


\title{
Texture features based microscopic image classification of liver cellular granuloma using artificial neural networks
}

\author{
Fuqian Shi ${ }^{1, *}$, Gaoxiang Chen ${ }^{1}$, Yu Wang ${ }^{1}$, Ningning Yang ${ }^{1}$, Yating Chen ${ }^{1}$, Nilanjan Dey ${ }^{2}$, and R. Simon Sherratt ${ }^{3}$ \\ 1. First Affiliated Hospital of Wenzhou Medical University, Wenzhou 325035, China. \\ 2. Department of Information Technology, Techno India College of Technology, West Bengal 740000, India \\ 3.Department of Biomedical Engineering, the University of Reading, RG6 6AY, UK. \\ \{sfq@wmu.edu.cn, 582372420@qq.com,710444803@qq.com,1062764811@qq.com,582372420@qq.com, \\ nilanjan.dey@tict.edu.in, r.s.sherratt@ reading.ac.uk\} \\ *Corresponding author: Fuqian Shi, sfq@wmu.edu.cn
}

\begin{abstract}
Automated classification of Schistosoma mansoni granulomatous microscopic images of mice liver using Artificial Intelligence (AI) technologies is a key issue for accurate diagnosis and treatment. In this paper, three grey difference statistics-based features, namely three Gray-Level Co-occurrence Matrix (GLCM) based features and fifteen Gray Gradient Co-occurrence Matrix (GGCM) features were calculated by correlative analysis. Ten features were selected for three-level cellular granuloma classification using a Scaled Conjugate Gradient BackPropagation Neural Network (SCG-BPNN) in the same performance. A cross-entropy is then calculated to evaluate the proposed Sigmoid input and the ten-hidden layer network. The results depicted that SCG-BPNN with texture features performs high recognition rate compared to using morphological features, such as shape, size, contour, thickness and other geometry-based features for the classification. The proposed method also has a high accuracy rate of $87.2 \%$ compared to the Back-Propagation Neural Network (BPNN), Back-Propagation Hopfield Neural Network (BPHNN) and Convolutional Neural Network (CNN).
\end{abstract}

Keywords: back-propagation neural network; gray gradient cooccurrence matrix; gray level co-occurrence matrix; microscopic image classification; scaled conjugate gradient

\section{INTRODUCTION}

Schistosoma mansoni granulomatous is a parasite egg deposited in a host leading to fibrosis diseases due to the mansoni infection. Granulomatous diseases of the liver span a huge range of infectious, drug-related, and immunologic disorders, which have different granulomas types and fibrosis stages [1]. Recently, researchers have conducted several experimental analyses on cellular granuloma of mice liver using high-resolution microscopes, such as cell phase-contrast microscopy and probe-based confocal laser endomicroscopy [2] [3]. Accordingly, image processing has a significant role to separate surrounding tissues based on shape, color and texture analysis to distinguish between normal and abnormal liver cases using the captured microscopic images for further diagnosis [4]. For automated computer-aided diagnosis systems, medical image processing, including image preprocessing, feature extraction and classification have all been developed.

For mice liver cellular granulomatous detection and classification, feature extraction has a significant role to measure and digitalize the inherent nature of the fibrosis regions and their attributes as well as to decompose and symbolize these regions to form the feature vector for further classification. Most researchers have focused on morphological feature based automatic recognition of microscopic images [5], while morphological feature calculations take more of a geometrical analysis on the microscopic images. On the contrary, texture analysis is an important research content for image understanding, analysis, recognition, and description of the difference of the structure, direction, granularity and regularity of the different regions of the microscopic image [6-7]. Moreover, texture contains not only the surface properties/characteristics but also some extent that reflects the relationship between them and the environment. Therefore, the texture features in gray statistical information can reflect the distribution structure and spatial information [8]. For diagnosis and treatment, the feature extraction process is followed by the classification process, where image quantitative analysis is used to calculate the texture of the Region of Interest (ROI) and morphological features, and form a feature vector space for the purposes of classification. In microscopic image classification, an artificial neural network is extensively used for prediction and classification. The artificial neural network has high-speed information processing ability and large knowledge storage capacity, and typical algorithms include the Back-Propagation Neural Network (BPNN), Competitive Kohonen Neural Network (CKNN), Feed-forward Neural Network (FNN) and Hopfield Neural Network (HNN). Hence, artificial neural networks have a significant role in medical image analysis and classification.

For microscopic liver image analysis and classification, Akram et al. [9] selected features based on texture and color 
properties for further classification of the abnormal liver images using the Support Vector Machine (SVM) to perform classification. The results depicted that the extracted texturebased features with the SVM classifier achieved an accuracy of $83 \%$ on a mice liver dataset. Furthermore, Amin and MahmoudGhoneim [10] applied statistical texture analysis approaches, namely run-length matrices and co-occurrence on microscopic images of rats' samples followed by classification using linear discriminant analysis and agglomerative hierarchical clustering. The classification results proved that texture analysis was successful to discriminate between the control and fibrosis groups with $100 \%$ sensitivity and specificity. Meng et al. [11] implemented texture features based histology image supervised classification and multimodal fusion. Xie et al. [12] used tumor color, texture and border features for melanoma classification; Kosmas Dimitropoulos et al. [13] used adaptive neuro-fuzzy inference systems for automatic detection of centroblasts $(\mathrm{CBs})$ in microscopic images.

Texture features calculation to find the defects of parameters, such as texture features that are specific to a pathological region has been shown to be difficult and time-consuming using BPNN based classification [14]. Therefore, it becomes necessary to develop techniques, such as conjugate gradient, to overcome this shortcoming. The conjugate gradient method is a special conjugate direction method, also related to the gradient, which utilizes the gradient information of an objective function (the product of the gradient and the direction satisfies the "descent" condition). The conjugate gradient method differs from that in the negative gradient direction of the current point and is conjugate with the search direction in front to obtain a new search direction [15 16]. Chen et al. [17] proposed a regularized deep feature extraction method using a Convolutional Neural Network (CNN) for hyperspectral image classification. Geng et al. [18] introduced deep supervised and contractive neural network using a Gray Level-Gradient Cooccurrence Matrix (GLGCM). Furthermore, Chen et al. [19] used BPNN for recognition on oceanic internal waves based on Gray Gradient Co-occurrence Matrix (GGCM). Yu used an improved LBP algorithm for texture and face classification [20].

In this paper, the gray level co-occurrence matrix (GLCM) is improved according to a weighting coefficient. The redundant features are removed through the analysis of the correlation of texture features of the microscopic image of Schistosoma mansoni based cellular granulomatous of mice liver. The graydifference based features, GLCM based features, combined with GGCM for cellular granuloma microscopic image of mice liver feature calculations is thus proposed in this paper. Furthermore, a scaled conjugate gradient BPNN (SCG-BPNN) for classification is deployed. The organization of the remaining sections is as follows. Section 2 presents the methods for image classification and the experimental process. Section 3 addresses the results and comparative analysis. Section 4 involves the conclusions of the present study.

\section{METHODOLOGY}

\section{A. Texture-based Features Extraction}

Statistical computation is carried out in regions containing multiple pixels and often has strong resistance to noise. In this work, 21 texture-based features were calculated, including grey difference statistics-based features (mean, contract, and entropy), GLCM (energy, correlation, and inertia moment) and GGCM based features (T1-T15). By correlative analysis, 10 features are then selected as a vector input for the Scaled Conjugate Gradient Back-Propagation Neural Network (SCGBPNN) [21].

Assume $(x, y)$ is a pixel's coordinates in a microscopic fibrosis image, where $x \in\{1,2, \ldots, m\}$, and $y \in\{1,2, \ldots, n\}$, which contains $L$ gray levels. Accordingly, $(x+\Delta x, y+\Delta y)$ is the nearby pixel, where $\Delta x>0$, and $\Delta y>0$ as $f(x, y)$ is the intensity value of this pixel. So, the gray value can be calculated by:

$$
g_{\Delta}(f(x, y))=g(f(x, y))-g(f(x+\Delta x, y+\Delta y))
$$

where $g_{\Delta}$ is the gray difference. Thus, for $m$ levels, by counting $g_{\Delta}$ in each $m$, the probability $p(i)$ on each $g_{\Delta}$ can be obtained, where $(i=1, \ldots, m)$.

(1) Gray Difference-based Features

Hence, the features of mean, contract, and entropy are given from [22]:

$$
\begin{gathered}
\text { mean }=\frac{1}{m} \sum_{i=1}^{m} i p(i) \\
\text { contract }=\sum_{i=0}^{m} i^{2} p(i) \\
\text { entropy }=-\sum_{i=0}^{m} p(i) \log _{2}(p(i))
\end{gathered}
$$

(2) Gray-level Co-occurrence Matrix-based Features

The GLCM is a common method to describe the texture by examining the spatial correlation characteristics of the gray level. It is based on the statistical analysis of two pixels with a given distance. Intuitively speaking, if the image is constituted with similar blocks of pixels' gray value, the GLCM is diagonal elements with relatively large value; if the pixel gray value change in the local area, then the off-diagonal elements will have relatively large values. The energy, correlation and inertia moment can be calculated as $[6,22,23]$ :

$$
\begin{gathered}
\text { energy }=\sum_{x=0}^{m-1} \sum_{y=0}^{m-1} P_{x y}^{2} \\
\text { correlation }=\frac{1}{\sigma_{X} \sigma_{Y}} \sum_{x=0}^{m-1} \sum_{y=0}^{m-1}\left(x-\mu_{X}\right)\left(y-\mu_{Y}\right) P_{x y} \\
i m=\sum_{i=0}^{L-1} \sum_{j=0}^{L-1}|i-j|^{2} P_{i j}
\end{gathered}
$$

where $P_{x y}$ is the probability of point $(x, y)$ in the $i=1, \cdots, m$ gray level, $\mu_{X}=\sum_{x=0}^{m-1} x \sum_{y=0}^{m-1} p_{x y} \quad, \quad \mu_{Y}=\sum_{y=0}^{m-1} y \sum_{x=0}^{m-1} p_{x y}$, 
$\sigma_{x}=\sqrt{\sum_{x=0}^{m-1}\left(x-\mu_{X}\right)^{2} \sum_{y=0}^{m-1} p_{x y}}$, and $\sigma_{y}=\sqrt{\sum_{y=0}^{m-1}\left(y-\mu_{Y}\right)^{2} \sum_{x=0}^{m-1} p_{x y}}$,

which are the variances and the standard deviations at both the $x$ and $y$ pixel location.

For rough texture, $P_{i j}$ are close to main diagonal, so that $i m$ is smaller, while for fine texture, im is larger.

(3) Gray Gradient Co-occurrence Matrix

The GGCM includes the extracted texture features by using gray and gradient synthetic information, which is similar in process to the GLCM. The Sobel operator may be applied to $f(x, y)$ and let $g(x, y)$ refer to gray that have the digitized form $G(x, y)$, thus the new gray $L_{g}$ is used to calculate the new gray image $G(x, y)$ as:

$$
G(x, y)=\frac{g(x, y)-\min (g(x, y))}{\max (g(x, y))-\min (g(x, y))}\left(L_{g}-1\right)
$$

The gray gradient co-occurrence matrix $H_{i j}$ is defined as number of $\{(x, y) \mid f(x, y)=0, G(x, y)=j\}$, which has the normalized form of:

$$
\left\|H_{i j}\right\|=\frac{H_{i j}}{\sum_{i=0}^{L-1} \sum_{j=0}^{L-1} H_{i j}}
$$

According to $\left\|H_{i j}\right\|, 15$ features are defined and extracted as reported in TABLE I [19]. The features mentioned above were then normalized and organized as an input vector for classification using SCG-BPNN, while the target used fewer features under the same performance by SCG-BPNN.

\section{B. Scaled Conjugate Gradient Back-propagation Neural Network}

The BPNN is a multilayer network with the Widrow-Hoff learning algorithm and nonlinear differentiable transfer function. A typical Back-Propagation (BP) network uses a gradient descent algorithm, defined by the Widrow-Hoff algorithm. It is a method for computing the gradient of a nonlinear multilayer network [24]. The input of the $J^{\text {th }}$ output layer unit is:

$$
\text { net } c_{J}=\sum_{J=1}^{P} W_{y} b_{J}
$$

where the actual output is given by:

$$
C_{J}=f\left(\text { netc }_{J}\right)
$$

The weighted input of the hidden layer unit $I$ is given by:

$$
\text { netb } b_{I}=\sum_{k=1}^{n} v_{y} a
$$

Thus, the actual output of the unit $I$ is:

$$
b_{I}=f\left(\text { net }_{I}\right)
$$

where $f$ is a differential decreasing function, given by:

$$
f(x)=\frac{1}{1+e^{-x}}
$$

The algorithm of these steps is described in Algorithm 1.

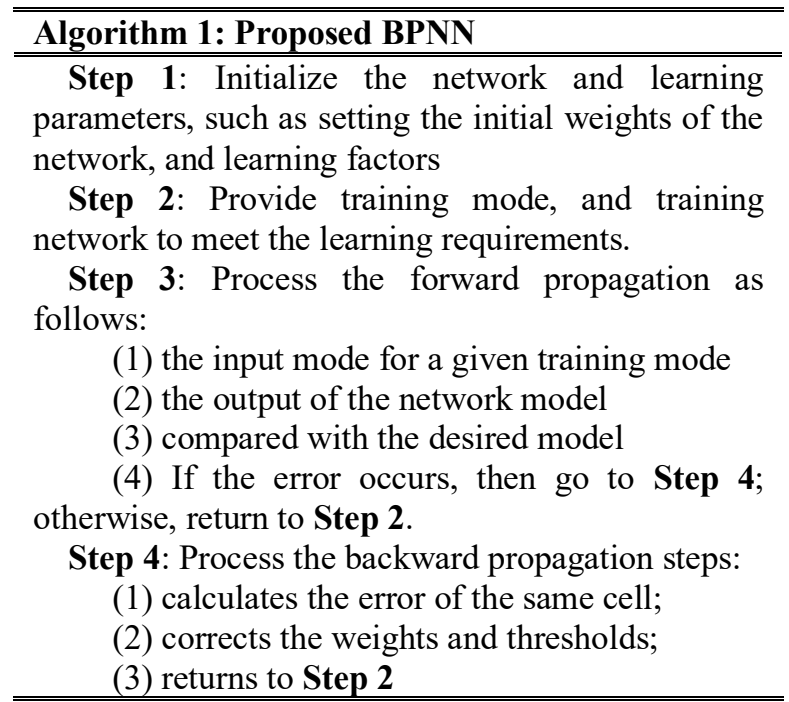

To guarantee fast/accurate convergence of the neural networks, the Scaled Conjugate Gradient (SCG) algorithm was applied. The conjugate gradient method uses the first derivative information, but overcomes the disadvantage of slow convergence of the steepest descent method. Additionally, it avoids the need to store and compute the Hesse matrix and the shortcomings of the inverse of the Newton method. It only requires a small storage capacity and provides fast convergence and high stability without any external parameters. However, the SCG uses second order information from the NN but requires only $O(N)$ memory usage, where $N$ is the number of weights in the network.

The performance of SCG was benchmarked against the performance of the standard BP, the Conjugate Gradient Backpropagation (CGB) algorithm and the one-step BroydenFletcher-Goldfarb-Shanno memoryless quasi-Newton algorithm (BFGS) [15]. The SCG process is described in Algorithm 2. The used pseudo-code is given in Algorithm 3.

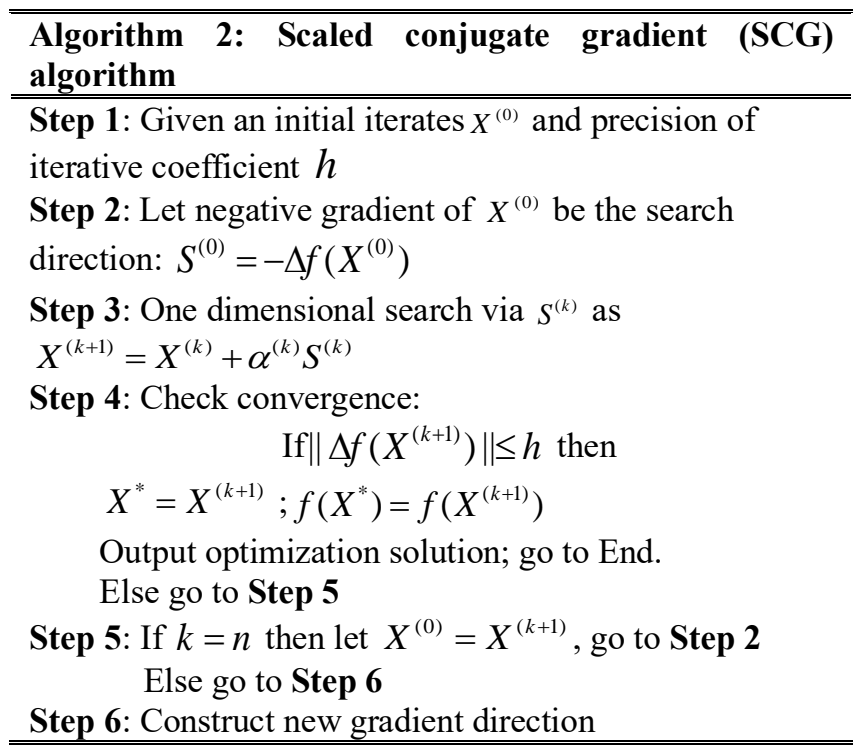




$$
\begin{aligned}
& \beta=\frac{\|\Delta f(x(k+1))\|^{2}}{\|\Delta f(X(k))\|^{2}} \\
& S^{(k+1)}=-\Delta f\left(X^{(k)}\right)+\beta^{(k)} S^{(k)} \\
& k=k+1 \text { go to Step 3 }
\end{aligned}
$$

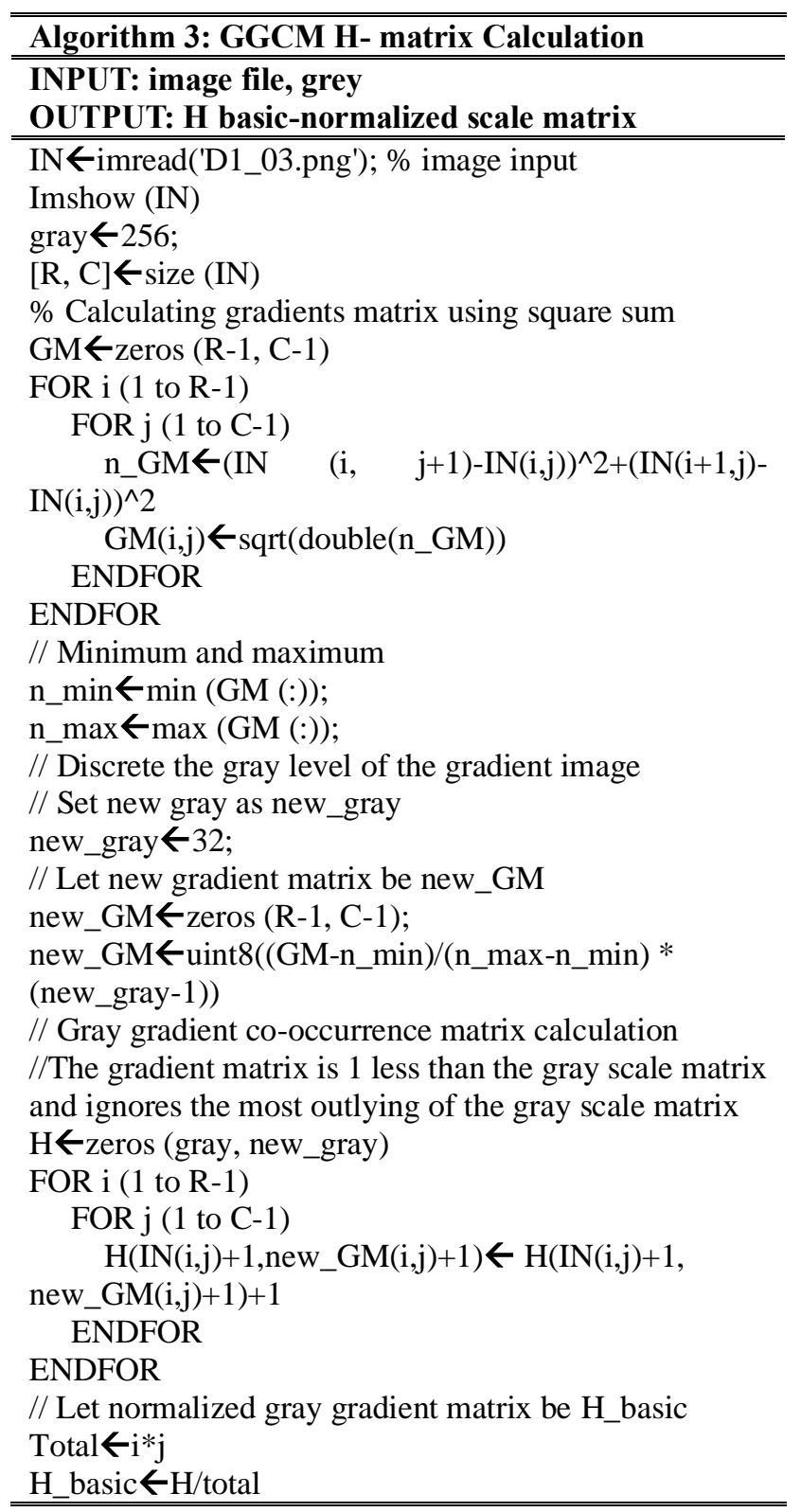

\section{RESULTS AND DISCUSSION}

In the current study, microscopic images of magnification 3400 were acquired and examined in the Medical Parasitology Department, Faculty of Medicine, Tanta University, Egypt. These acquired liver samples from normal and Schistosomiasis mansoin infected mice are illustrated in Fig. 1. Some representative images of histopathological samples are obtained from the control liver group; fibrosis and granuloma are illustrated in Fig. 1(a)-(c). Fig. 1(a) demonstrated that the histological analysis of healthy liver shows a normal liver lobular architecture, while the liver fibrosis in Fig. 1(b) has disruption of the tissue architecture, the extension of fibers, and fibers accumulation. In addition, the liver granuloma in Fig. 1(c) shows multinucleated giant cells and lymphocytes or a conglomeration of smaller granulomas. Images were encoded at 24-bits per pixel on red, green, and blue channels.

The colored images were processed using Matlab (version 2017a) to create grey level images of size $256 * 3 * 256$ and to compute the gradient vectors matrices. To increase the dataset size, each healthy liver image was divided into two sub-images of $256 * 3 * 128$ size. Thus, 20 images of each class were used in this study. The training phase used 30 images ( 10 of each class) and the test phase used the rest of 30 images.

\section{A. Features Calculation}

Mean (M), Contract (Co), Entropy (E), Energy (En), Correlation (C), and Inertia Moment (IM) of 30 images were calculated and shown in Table 1. The four directions of 0,45 , 90, and 135 on En, C, and IM were calculated and the mean of these four directions was adopted for the next step. The GGCM features in Tables 2-4 list the 1st, 2nd, and 3rd level-fibro cellular granuloma.

\section{B. Training process}

A ten-hidden layer NN design with three grey difference statistics-based feature (M, Co, E), three gray-level cooccurrence matrix-based features (En, C, IM), and gray gradient co-occurrence matrix-based features (T1-T15) was constructed. There were 21 features of images, 3200 data applied. Thus, the input matrix is $21 * 3200$, and the target matrix is $3 * 3200$, in which $1^{\text {st }}$ level-fibro cellular granuloma is $[1,0,0]^{T}, 2^{\text {nd }}$ levelfibro cellular granuloma is $[0,1,0]^{T}$, and the $3^{\text {rd }}$ level-fibro cellular granuloma is $[0,0,1]^{T}$. The cross-entropy was performed with the proposed SCG-BPNN training process as shown in Fig. 2. Setting $70 \%$ of data for training (2240 records), was presented to the network during training, and the network was adjusted according to its error; $15 \%$ for validation (480 records), which is used to measure network generalization, and to halt training when generalization stops improving; and $15 \%$ for testing (480 records), which is to provide an independent measure of network performance during and after training as shown in Fig. 3 [25].

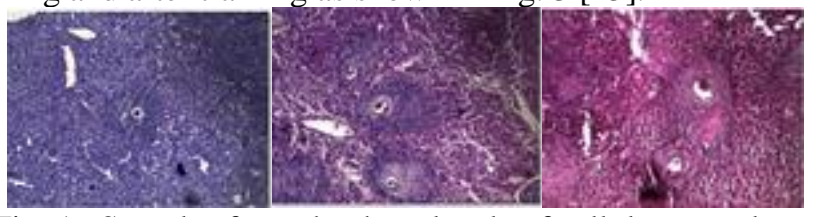

Fig. 1. Samples from the three levels of cellular granuloma microscopic images of mice liver, where (a) first level-fibro cellular granuloma, (b) second level-fibro cellular granuloma, and (c) third level-fibro cellular granuloma.

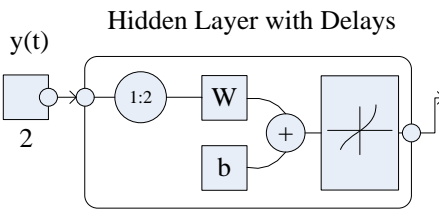

10
Output Layer

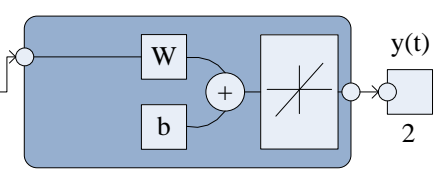

2
Fig. 2. 10 hidden layers BPNN using SCG. 
Table 1 GLCM Features' value of three classifications

\begin{tabular}{|c|c|c|c|c|c|c|}
\hline Images & $\operatorname{Mean}(\mathrm{M})$ & Contract $(\mathrm{Co})$ & Entropy (E) & Energy (En) & Correlation (C) & $\begin{array}{l}\text { Inertia Moment } \\
\text { (IM) }\end{array}$ \\
\hline A1 & 0.5846 & $2.27 \mathrm{E}+04$ & 5.9544 & 0.0205 & 0.8963 & 2.5124 \\
\hline A2 & 0.549 & $2.01 \mathrm{E}+04$ & 5.9662 & 0.0214 & 0.9061 & 3.5518 \\
\hline A3 & 0.5728 & $2.18 \mathrm{E}+04$ & 5.9897 & 0.0201 & 0.8857 & 2.8477 \\
\hline A4 & 0.5474 & $2.00 \mathrm{E}+04$ & 5.9863 & 0.0187 & 0.9063 & 3.2446 \\
\hline A5 & 0.5516 & $2.01 \mathrm{E}+04$ & 5.2921 & 0.0443 & 0.9440 & 0.8211 \\
\hline A6 & 0.5508 & $2.01 \mathrm{E}+04$ & 5.5094 & 0.0344 & 0.9400 & 1.9194 \\
\hline A7 & 0.5935 & $2.33 \mathrm{E}+04$ & 5.6296 & 0.0359 & 0.9148 & 1.3437 \\
\hline $\mathrm{A} 8$ & 0.6669 & $2.92 \mathrm{E}+04$ & 4.8391 & 0.0453 & 0.9684 & 0.5520 \\
\hline A9 & 0.5909 & $2.30 \mathrm{E}+04$ & 5.3227 & 0.0359 & 0.9404 & 1.2386 \\
\hline A10 & 0.5029 & $1.68 \mathrm{E}+04$ & 5.6044 & 0.0263 & 0.9174 & 2.7570 \\
\hline $\mathrm{B} 1$ & 0.5737 & $2.18 \mathrm{E}+04$ & 5.8114 & 0.0223 & 0.9184 & 2.4489 \\
\hline $\mathrm{B} 2$ & 0.5116 & $1.73 \mathrm{E}+04$ & 5.1946 & 0.0353 & 0.9442 & 1.1807 \\
\hline B3 & 0.5505 & $2.01 \mathrm{E}+04$ & 5.5709 & 0.0245 & 0.9296 & 1.9294 \\
\hline B4 & 0.5551 & $2.04 \mathrm{E}+04$ & 5.7232 & 0.0219 & 0.9124 & 2.4392 \\
\hline B5 & 0.5675 & $2.13 \mathrm{E}+04$ & 5.4361 & 0.0264 & 0.9358 & 1.6751 \\
\hline B6 & 0.5775 & $2.20 \mathrm{E}+04$ & 5.6042 & 0.0284 & 0.9194 & 1.7089 \\
\hline B7 & 0.5741 & $2.18 \mathrm{E}+04$ & 5.7882 & 0.0216 & 0.9148 & 2.2714 \\
\hline B8 & 0.5574 & $2.06 \mathrm{E}+04$ & 5.5938 & 0.0276 & 0.9242 & 2.3149 \\
\hline B9 & 0.5567 & $2.06 \mathrm{E}+04$ & 5.7916 & 0.0202 & 0.8831 & 3.3948 \\
\hline B10 & 0.5366 & $1.91 \mathrm{E}+04$ & 5.6746 & 0.0240 & 0.9223 & 2.6020 \\
\hline $\mathrm{C} 1$ & 0.5103 & $1.76 \mathrm{E}+04$ & 6.4062 & 0.0176 & 0.7574 & 5.5554 \\
\hline $\mathrm{C} 2$ & 0.4928 & $1.64 \mathrm{E}+04$ & 6.2778 & 0.0209 & 0.7753 & 4.9723 \\
\hline $\mathrm{C} 3$ & 0.4978 & $1.69 \mathrm{E}+04$ & 6.5513 & 0.0162 & 0.7270 & 6.2586 \\
\hline $\mathrm{C} 4$ & 0.5263 & $1.87 \mathrm{E}+04$ & 6.3818 & 0.0149 & 0.7163 & 5.3177 \\
\hline $\mathrm{C} 5$ & 0.5113 & $1.78 \mathrm{E}+04$ & 6.5959 & 0.0145 & 0.6927 & 7.2491 \\
\hline $\mathrm{C} 6$ & 0.5217 & $1.83 \mathrm{E}+04$ & 6.3612 & 0.0197 & 0.7039 & 5.3325 \\
\hline $\mathrm{C} 7$ & 0.5216 & $1.82 \mathrm{E}+04$ & 6.0952 & 0.0247 & 0.8114 & 3.8107 \\
\hline $\mathrm{C} 8$ & 0.4977 & $1.69 \mathrm{E}+04$ & 6.5391 & 0.0164 & 0.6755 & 6.9137 \\
\hline $\mathrm{C} 9$ & 0.5084 & $1.75 \mathrm{E}+04$ & 6.3609 & 0.0194 & 0.7469 & 5.0677 \\
\hline $\mathrm{C} 10$ & 0.4994 & $1.70 \mathrm{E}+04$ & 6.5444 & 0.0149 & 0.7167 & 5.1681 \\
\hline
\end{tabular}

Table 2. 1st level-fibro cellular granuloma

\begin{tabular}{|c|c|c|c|c|c|c|c|c|c|c|}
\hline $\mathrm{T} 1$ & $\mathrm{~A} 1$ & $\mathrm{~A} 2$ & A3 & A4 & A5 & A6 & A7 & A8 & A9 & A10 \\
\hline $\mathrm{T} 1$ & 0.3019 & 0.3139 & 0.3024 & 0.3152 & 0.3294 & 0.3257 & 0.3141 & 0.3364 & 0.3248 & 0.3390 \\
\hline $\mathrm{T} 2$ & 15.5918 & 15.3242 & 15.6748 & 14.6702 & 10.7114 & 12.3308 & 12.9371 & 9.2091 & 12.1174 & 13.3615 \\
\hline $\mathrm{T} 3$ & 48666.9506 & 60813.9609 & 48886.0521 & 53214.4055 & 60096.4041 & 53995.5007 & 59367.6702 & 51247.4335 & 54294.4579 & 73663.4528 \\
\hline $\mathrm{T} 4$ & 1912331.3988 & 2016587.3570 & 1950295.7037 & 1879498.2690 & 1302821.3737 & 1488417.5935 & 1433212.6591 & 1282583.9918 & 1419916.1481 & 1752166.1768 \\
\hline $\mathrm{T} 5$ & 0.0011 & 0.0026 & 0.0011 & 0.0018 & 0.0009 & 0.0011 & 0.0009 & 0.0008 & 0.0009 & 0.0035 \\
\hline T6 & 105.5963 & 114.8666 & 103.9456 & 109.8143 & 101.2361 & 98.2116 & 96.8497 & 115.6000 & 105.9825 & 117.0239 \\
\hline $\mathrm{T} 7$ & 15.5918 & 15.3242 & 15.6748 & 14.6702 & 10.7114 & 12.3308 & 12.9371 & 9.2091 & 12.1174 & 13.3615 \\
\hline $\mathrm{T} 8$ & 57.2349 & 69.7490 & 58.3750 & 68.3788 & 45.4182 & 59.3752 & 46.3586 & 52.3749 & 53.7958 & 71.3283 \\
\hline T9 & 13.2476 & 13.4091 & 13.2915 & 13.2480 & 11.1861 & 12.2821 & 12.2932 & 10.1674 & 12.0661 & 12.9066 \\
\hline $\mathrm{T} 10$ & -111.5312 & -206.1009 & -123.9672 & -104.1345 & -9.1072 & -3.0095 & -44.2040 & -50.1271 & -10.8259 & -155.0087 \\
\hline $\mathrm{T} 11$ & 2.3345 & 2.3310 & 2.3326 & 2.3464 & 2.2555 & 2.3066 & 2.2602 & 2.3081 & 2.2974 & 2.3194 \\
\hline $\mathrm{T} 12$ & 0.9691 & 0.9452 & 0.9604 & 0.9744 & 1.1261 & 1.0684 & 1.0835 & 1.1341 & 1.0891 & 1.0085 \\
\hline $\mathrm{T} 13$ & 3.2876 & 3.2281 & 3.2747 & 3.2829 & 3.3727 & 3.3461 & 3.3356 & 3.4238 & 3.3682 & 3.2780 \\
\hline $\mathrm{T} 14$ & 11775.2088 & 15365.6265 & 11623.9618 & 14111.8418 & 10364.4484 & 11057.7967 & 9429.9789 & 14065.2696 & 11871.8984 & 16310.2245 \\
\hline $\mathrm{T} 15$ & 0.0083 & 0.0109 & 0.0095 & 0.0131 & 0.0029 & 0.0076 & 0.0052 & 0.0012 & 0.0037 & 0.0106 \\
\hline $\mathrm{T} 1$ & B1 & B2 & B3 & B4 & B5 & B6 & B7 & B8 & B9 & B10 \\
\hline $\mathrm{T} 1$ & 0.3086 & 0.3142 & 0.3199 & 0.3102 & 0.3150 & 0.3131 & 0.3054 & 0.3293 & 0.3246 & 0.3314 \\
\hline $\mathrm{T} 2$ & 14.2179 & 12.1362 & 13.1355 & 14.0665 & 12.8627 & 13.5024 & 14.2457 & 13.2809 & 14.1569 & 13.7770 \\
\hline $\mathrm{T} 3$ & 47471.7426 & 52280.3008 & 51809.5428 & 48676.1086 & 52538.3466 & 52287.9610 & 47559.9637 & 59497.6592 & 57677.1244 & 64358.5400 \\
\hline $\mathrm{T} 4$ & 1690970.5634 & 1355140.5665 & 1536245.5592 & 1649390.1181 & 1437381.0482 & 1523692.4917 & 1637523.8702 & 1646636.8041 & 1771055.6159 & 1780784.7645 \\
\hline $\mathrm{T} 5$ & 0.0010 & 0.0009 & 0.0013 & 0.0011 & 0.0011 & 0.0009 & 0.0009 & 0.0021 & 0.0021 & 0.0028 \\
\hline T6 & 105.2023 & 104.3330 & 114.8417 & 106.4662 & 105.0355 & 102.4853 & 105.1254 & 106.3970 & 116.4003 & 115.0945 \\
\hline $\mathrm{T} 7$ & 14.2179 & 12.1362 & 13.1355 & 14.0665 & 12.8627 & 13.5024 & 14.2457 & 13.2809 & 14.1569 & 13.7770 \\
\hline $\mathrm{T} 8$ & 62.1734 & 58.1669 & 61.5291 & 61.1262 & 58.5088 & 53.8183 & 59.3140 & 67.2009 & 65.6223 & 69.0537 \\
\hline T9 & 12.9438 & 11.9675 & 12.5391 & 12.8662 & 12.2912 & 12.5619 & 12.8598 & 12.7302 & 13.0260 & 13.0237 \\
\hline $\mathrm{T} 10$ & -51.0151 & -20.1204 & -48.5817 & -56.4202 & -44.0639 & -25.8506 & -60.6437 & -92.3635 & -127.3191 & -130.2682 \\
\hline $\mathrm{T} 11$ & 2.3454 & 2.3147 & 2.3329 & 2.3392 & 2.3152 & 2.3102 & 2.3408 & 2.3217 & 2.3324 & 2.3277 \\
\hline
\end{tabular}




\begin{tabular}{|c|c|c|c|c|c|c|c|c|c|c|}
\hline $\mathrm{T} 12$ & 1.0162 & 1.1017 & 1.0574 & 1.0280 & 1.0810 & 1.0604 & 1.0299 & 1.0316 & 1.0025 & 0.9988 \\
\hline $\mathrm{T} 13$ & 3.3391 & 3.3880 & 3.3610 & 3.3432 & 3.3731 & 3.3571 & 3.3522 & 3.3137 & 3.2961 & 3.2809 \\
\hline $\mathrm{T} 14$ & 12413.2483 & 11986.6066 & 14384.3877 & 12552.4968 & 12158.3096 & 11023.8733 & 12063.9336 & 13533.3587 & 15184.3276 & 15463.8116 \\
\hline T15 & 0.0091 & 0.0050 & 0.0052 & 0.0086 & 0.0066 & 0.0059 & 0.0083 & 0.0109 & 0.0076 & 0.0093 \\
\hline $\mathrm{T} 1$ & $\mathrm{C} 1$ & $\mathrm{C} 2$ & $\mathrm{C} 3$ & $\mathrm{C} 4$ & $\mathrm{C} 5$ & C6 & $\mathrm{C} 7$ & $\mathrm{C} 8$ & $\mathrm{C} 9$ & $\mathrm{C} 10$ \\
\hline $\mathrm{T} 1$ & 0.3111 & 0.3168 & 0.3101 & 0.2995 & 0.3037 & 0.3068 & 0.3066 & 0.3002 & 0.3022 & 0.2985 \\
\hline $\mathrm{T} 2$ & 16.7161 & 16.1987 & 17.3973 & 17.1418 & 17.5933 & 16.8010 & 15.3368 & 17.5719 & 16.8337 & 17.4637 \\
\hline $\mathrm{T} 3$ & 9173.0569 & 9215.6099 & 9269.3695 & 7711.2967 & 7637.8552 & 8235.4866 & 10043.9520 & 7975.3021 & 9375.5377 & 6902.9449 \\
\hline $\mathrm{T} 4$ & 419324.6869 & 370394.6909 & 450648.6879 & 350891.7553 & 411413.7763 & 365925.4825 & 353987.2040 & 413205.5556 & 428587.4277 & 359326.2402 \\
\hline $\mathrm{T} 5$ & 0.0021 & 0.0023 & 0.0025 & 0.0015 & 0.0021 & 0.0015 & 0.0014 & 0.0017 & 0.0015 & 0.0016 \\
\hline T6 & 110.5868 & 110.2067 & 116.5026 & 94.5674 & 111.8048 & 94.4182 & 99.4258 & 100.8519 & 99.9060 & 119.8363 \\
\hline $\mathrm{T} 7$ & 16.7161 & 16.1987 & 17.3973 & 17.1418 & 17.5933 & 16.8010 & 15.3368 & 17.5719 & 16.8337 & 17.4637 \\
\hline $\mathrm{T} 8$ & 63.2031 & 63.5937 & 63.2421 & 53.1304 & 62.1079 & 54.4959 & 58.5130 & 58.1464 & 57.8339 & 52.7525 \\
\hline T9 & 13.7180 & 13.6377 & 13.8599 & 13.6579 & 13.8366 & 13.6584 & 13.2450 & 13.7858 & 13.6211 & 13.7487 \\
\hline $\mathrm{T} 10$ & -191.5271 & -208.2920 & -233.2392 & -156.7994 & -196.6382 & -139.1340 & -101.1759 & -177.5633 & -133.8385 & -178.7957 \\
\hline $\mathrm{T} 11$ & 2.3314 & 2.3186 & 2.3295 & 2.3024 & 2.3376 & 2.2991 & 2.3055 & 2.3193 & 2.3176 & 2.3199 \\
\hline $\mathrm{T} 12$ & 0.8608 & 0.8897 & 0.8072 & 0.8580 & 0.8022 & 0.8681 & 0.9699 & 0.8148 & 0.8761 & 0.8268 \\
\hline $\mathrm{T} 13$ & 3.1541 & 3.1664 & 3.0936 & 3.1414 & 3.1029 & 3.1432 & 3.2454 & 3.1049 & 3.1672 & 3.1246 \\
\hline $\mathrm{T} 14$ & 13377.5885 & 13484.2243 & 14479.9928 & 9317.7039 & 13317.9315 & 9459.0545 & 10872.5308 & 10861.7394 & 10698.9772 & 13809.5914 \\
\hline $\mathrm{T} 15$ & 0.0090 & 0.0090 & 0.0077 & 0.0106 & 0.0085 & 0.0108 & 0.0098 & 0.0101 & 0.0100 & 0.0047 \\
\hline
\end{tabular}

Table 5. Predictive accuracy rate with different neural networks

\begin{tabular}{llll}
\hline \hline T1 & CE & MSE & PAR \\
\hline BPNN [21] & 0.62 & 0.077 & 0.855 \\
CNN [27] & 0.71 & 0.068 & 0.788 \\
BPHNN [23] & 0.61 & 0.066 & 0.685 \\
FUZZY NN [28] & 0.65 & 0.082 & 0.752 \\
SCG-BPNN-10 & 0.67 & 0.066 & 0.872 \\
SCG-BPNN-21 & 0.75 & 0.071 & 0.821 \\
\hline \hline
\end{tabular}

Table 6. Comparing to time consuming in large scale dataset (70\% for training)

\begin{tabular}{lll}
\hline \hline Samples & SCG-BPNN-10 & SCG-BPNN-21 \\
\hline 1020 & 0.077 & 0.62 \\
2050 & 0.068 & 0.71 \\
3600 & 0.066 & 0.61 \\
5400 & 0.082 & 0.65 \\
7200 & 0.071 & 0.75 \\
1020 & 0.066 & 0.67 \\
\hline \hline
\end{tabular}

By correlative analysis of the features' calculations, especially for texture-based features, M, Co, E, C, IM and T2, T4, T6, T7, T15 were selected as a vector input for the SCGBPNN by correlative analysis, where the results are illustrated in Fig. 4. The results establish the superiority of the proposed features with the SCG-BPNN classifier. Additionally, the prediction result is very important for $\mathrm{NN}$ models. The learning process in fact is to adjust system parameters to make the results more and more accurate, which are the accuracy rate, is to accumulate the "loss" of each sample, and then calculate the average value [24-25].

A comparative study was performed with other related studies using the BPNN, CNN, BPHNN, and fuzzy NN with the proposed methods SGC-BPNN-10 and SGC-BPNN-21. All features are reported in Table 5 showing the Predictive Accuracy Rate (PAR), Cross Entropy (CE) and Mean Squared Error (MSE). The different algorithms listed in TABLE V were used for the same dataset from Section 3.2. It is obvious that the proposed method SCG-BPNN-10 has higher PAR with low
MSE. Furthermore, the SCG-BNPP-21 took higher computation time compared to the SCG-BPNN-10 as demonstrated in Table 6.
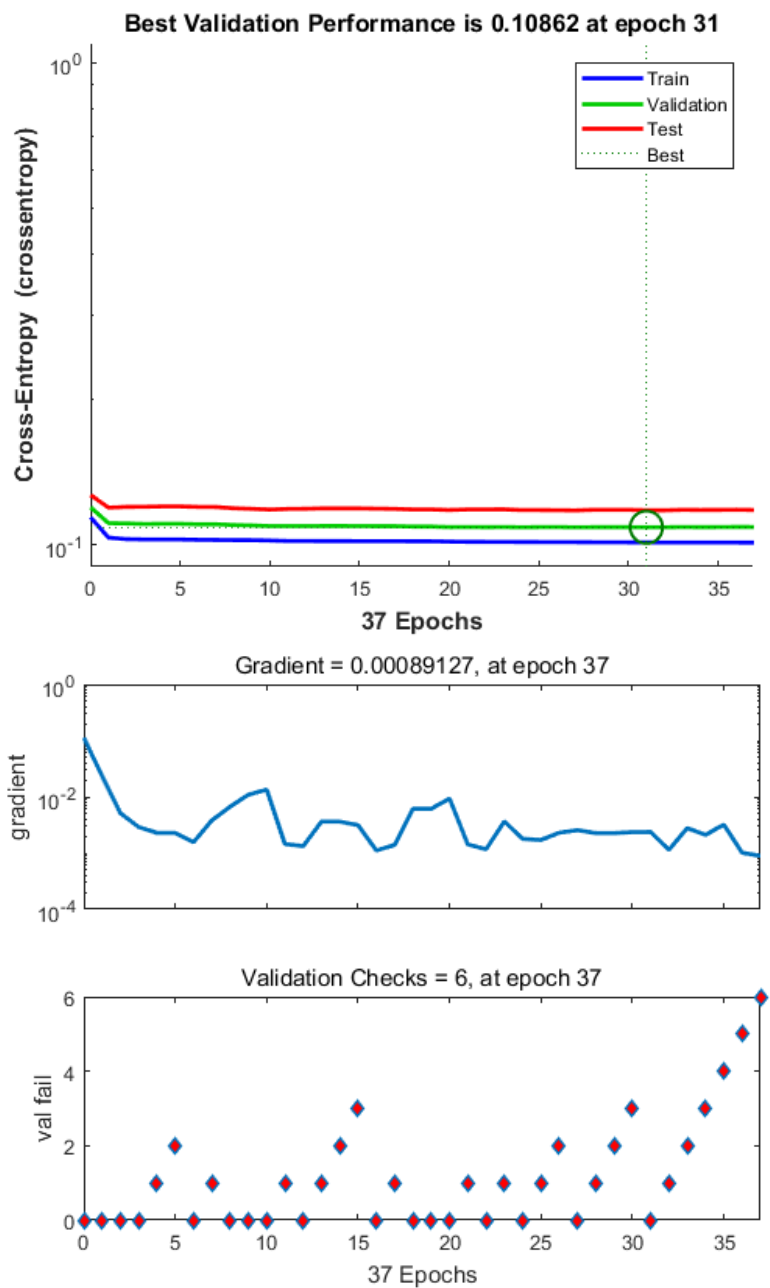

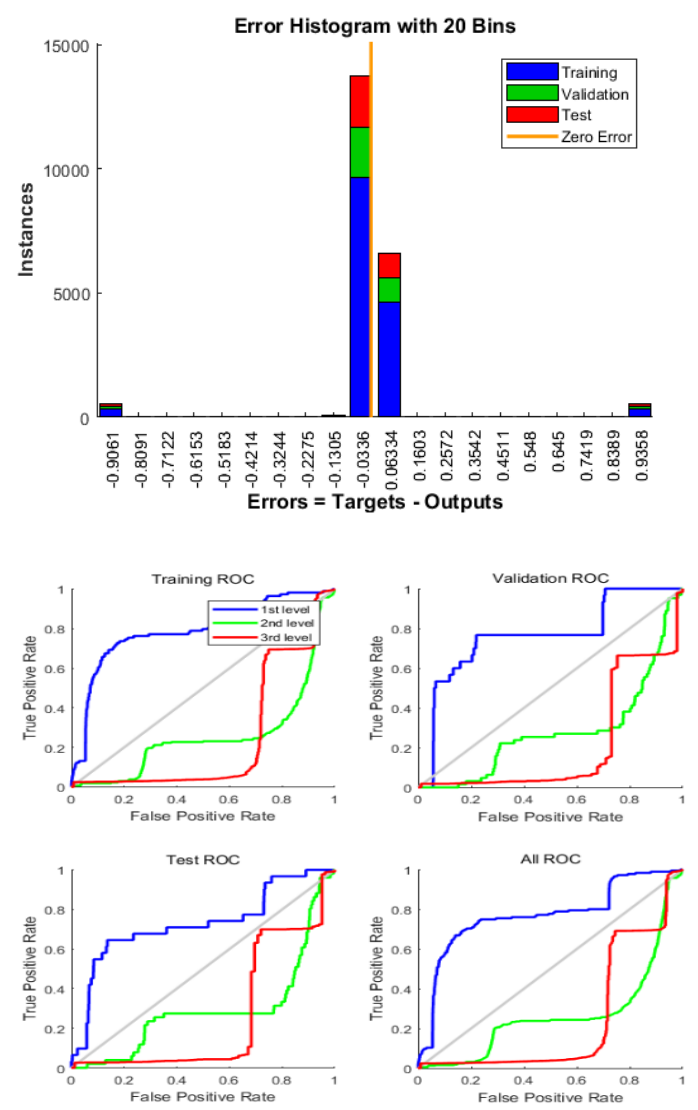

Fig. 3. Performance by epochs, training state, error histogram and receiver operating characteristic with all features.
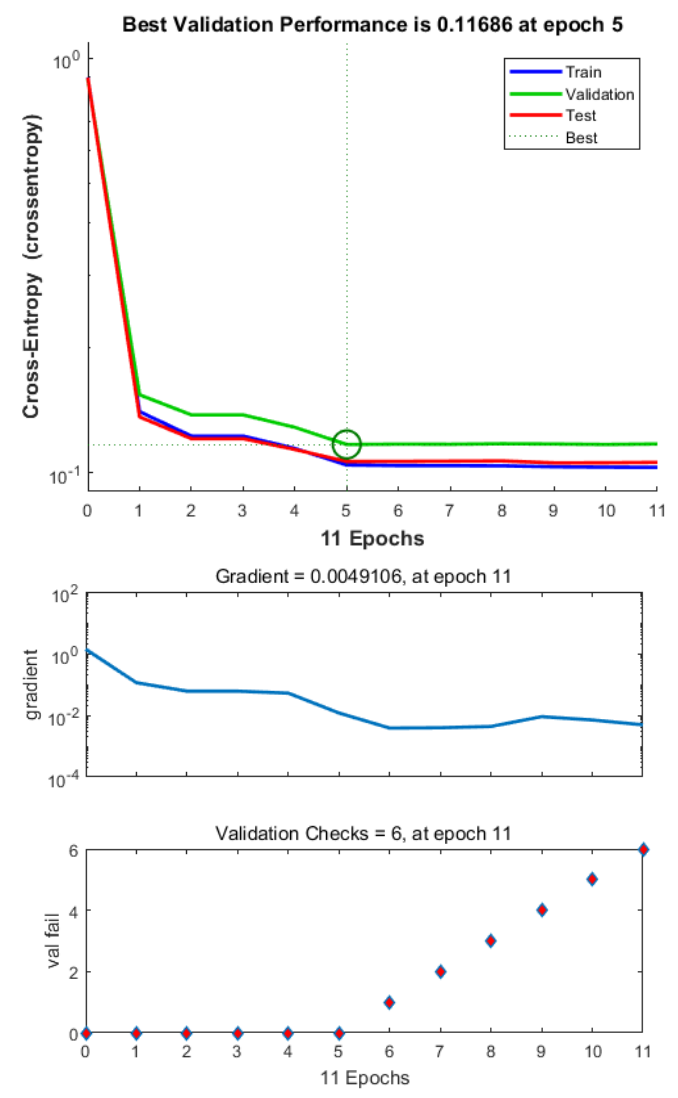
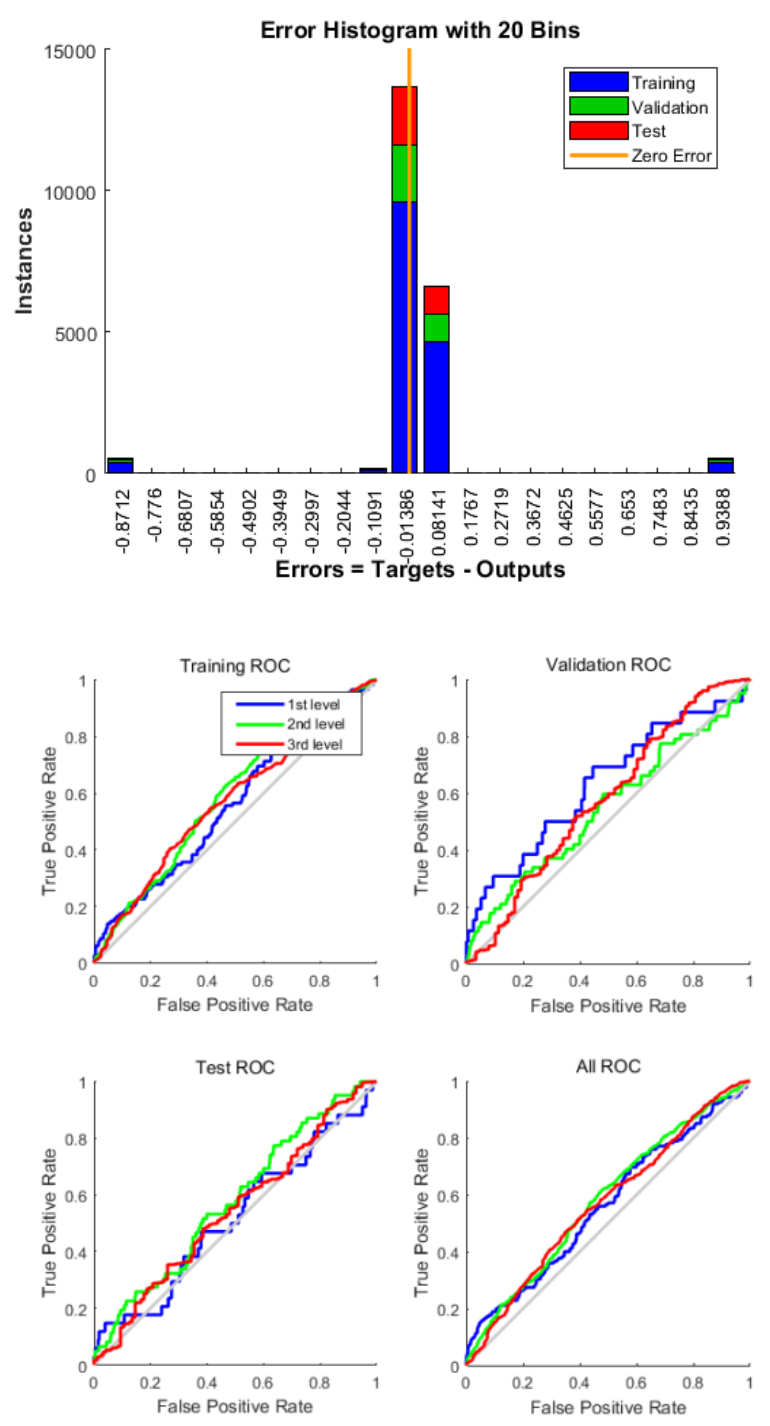

Fig. 4. Performance by epochs, training state, error histogram and receiver operating characteristic with ten features after correlative analysis.

\section{CONCLUSIONS}

Pathologists primarily use microscopic methods to qualitatively analyze microscopic images of cancer cells by using visual methods. The use of empirical knowledge to diagnose people's health has a significant role in clinical pathology. However, such methods are subjective and consume significant time. Furthermore, the captured microscopic images may be blurred, noisy, have poor contrast, unclear boundary, and human visual fatigue caused by long-term reading. These drawbacks will affect the accuracy of diagnostic analysis and judgment. With the rapid development of computer technology and the maturity of image graphics processing technology, the requirements for detecting objectivity are getting higher and higher, and computer image processing and analysis technology plays an increasingly important role in clinical diagnosis and treatment. The traditional method is to explore the classification, cell counting and texture analysis of precancerous lesions, pathology image retrieval and 
management, visualization surgery and reconstruction of human body models. This paper diagnoses diseases by identifying and analyzing microscopic images of diseased cells and can accurately identify and classify microscopic images [26]. The results are as follows:

(1) Realizing the automatic classification of microscopic images of Schistosoma mansoni granuloma using AI technology,

(2) Texture features such as CGCM and GGCM are calculated, and the SCG-BPNN based network training has been verified.

(3) Through cross entropy evaluation, Sigmod input and ten hidden layer network, the training results show that our SCGBPNN-10 has a high recognition rate with texture features. Compared with BPNN, back-propagation BPHNN and CNN, the proposed method also has higher accuracy.

In the future, insufficient training data, the design of neural network structures, and the definition of loss functions will all be considered.

\section{ACKNOWLEDGMENT}

This work is supported by Zhejiang Provincial Natural Science Foundation (Grant no: LY17F030014). Dalia S. Ashour, Assistant Professor of Medical Parasitology and Dina M. Aboraya, Lecturer of Medical Parasitology, Medical Parasitology Department, Faculty of Medicine, Tanta University, Egypt perfumed microscopic experiment and preprocessing the dataset.

\section{REFERENCES}

[1] D. E. Kleiner, "Granulomas in the liver," Seminars in Diagnostic Pathology, vol.23, no.3-4, pp.161-169, 2006.

[2] C. H. Huang, S. Sankaran, D. Racoceanu, S. Hariharan, and Sohail Ahmed "Online 3-D tracking of suspension living cells imaged with phasecontrast microscopy," IEEE Trans. Biomed. Eng., vol. 59, no.7, pp.19241933, 2012.

[3] W. T. Latt, R. C. Newton, V.S. Marco, C. J. Payne, D. P. Noonan, J. Z Shang, and G. Z. Yang, "A hand-held instrument to maintain steady tissue contact during probe-based confocal laser endomicroscopy," IEEE Trans. Biomed. Eng., vol. 58, no.9, pp.2694-2703, 2011.

[4] F. Zhao, X. X. Li, S. Biswas, R. Mullick, P. R. S. Mendonca and V. Vaidya, "Topological texture-based method for mass detection in breast ultrasound image," in Proc. ISBI, Beijing, China, 2014, pp. 685-689.

[5] J.-P. Thiran, and B. Macq, "Morphological feature extraction for the classification of digital images of cancerous tissues," IEEE Trans. Biomed. Eng., vol.43, no.10, pp.1011-1020, 1996.

[6] D. Choudhury, A. Naug, and S. Ghosh, "Texture and color feature based WLS framework aided skin cancer classification using MSVM and ELM," in Proc. INDICON, New Delhi, India, 2015.

[7] M. Havaei, A. Davy, W. F. David, A. Biard, A. Courville, Y. Bengio, C. Pal, P. M. Jodoin, H. Larochelle, "Brain tumor segmentation with Deep Neural Networks," Medical Image Analysis, vol. 35, pp.18-31, 2017.

[8] D. Schwenninger, K. Moller, H. Liu, and J. Guttmann, "Automated analysis of intratidal dynamics of alveolar geometry from microscopic endoscopy," IEEE Trans. Biomed. Eng., vol. 57, no.2, pp.415-421, 2010.

[9] M. U. Akram, M. A. Khan, M. Naveed, and S. Gul, "Liver image analysis using color and texture descriptors," in Int. Conf. Digital Information Processing, Electronics, and Wireless Communications (DIPEWC2016), Dubai, United Arab Emirates, 2016.

[10] A. Amin, and D. Mahmoud-Ghoneim, "Texture analysis of liver fibrosis microscopic images: a study on the effect of biomarkers," Acta Biochim Biophys Sin, vol. 43, no.3, pp.193-203, 2011.

[11] T. Meng, L. Lin, M. L. Shyu, and S. C. Chen, "Histology image classification using supervised classification and multimodal fusion," in Proc. ISM, Taichung, Taiwan, pp. 145-152, 2010.
[12] F. Y. Xie, H. D. Fan, Y. Li, Z. G. Jiang, R. S. Meng, and A. Bovik, "Melanoma classification on dermoscopy images using a neural network ensemble model," IEEE Trans. Med. Imag., vol. 36, no.3, pp. 849-858, 2017.

[13] Kosmas DimitropoulosEmmanouil MichailTriantafyllia KoletsaIoannis KostopoulosNikos Grammalidis, Using adaptive neuro-fuzzy inference systems for the detection of centroblasts in microscopic images of follicular lymphoma, Signal, Image and Video Processing, vol. 8, pp. 3340, 2014.

[14] G. Mohan, R. Karthik, P. Swathi Lakshmi, and P. Sajida, "A combinational network for high density noise removal and texture classification using back propagation network," Materials Today: Proceedings, vol. 4, no.8, pp. 7770-7777, 2017.

[15] C. S. Jiang, H. B. Li, and M. Rangaswamy, "On the conjugate gradient matched filter," IEEE Trans. Signal Process., vol. 60, no.5, pp. 2660-2666, 2012.

[16] F. Lenti, F. Nunziata, C. Estatico and M. Migliaccio, "Conjugate gradient method in Hilbert and Banach spaces to enhance the spatial resolution of radiometer Data," IEEE Trans. Geosci. Remote Sens., vol. 54, no.1, pp.397-406, 2016.

[17] Y. S. Chen, H. L. Jiang, C. Y. Li, X.P. Jia, and P. Ghamisi, "Deep feature extraction and classification of hyperspectral images based on convolutional neural networks," IEEE Trans. Geosci. Remote Sens., vol. 54, no.10, pp. 6232-6251, 2016.

[18] J. Geng, H. Y. Wang, J. C. Fan, and X. R. Ma, "Deep supervised and contractive neural network for SAR image classification," IEEE Trans. Geosci. Remote Sens., vol. 55, no. 4, pp. 2442-2459, 2017.

[19] F.-F. Chen, X.-F. Jiang, and Z.-Y. Jiang, "The research of recognition on oceanic internal waves based on gray gradient co-occurrence matrix and BP neural network," in Proc. SOPO, Wuhan, China, 2011.

[20] Wei Yu, Lin Gan, Sha Yang, Yonggang Ding, Pan Jiang, Jun Wang, Shijun Li, An improved LBP algorithm for texture and face classification, vol. 8, pp. 155-161, 2014.

[21] K. R. Singh, and S. Chaudhury, "Efficient technique for rice grain classification using back-propagation neural network and wavelet decomposition," IET Computer Vision, vol. 10, no. 8, pp. 780-787, 2016.

[22] R. M. Haralick, K Shanmugam, and I. Dinstein, "Textural features for image classification," IEEE Trans Syst., Man, and Cybern., vol. 3, no. 6, pp. 610-621, 1973 .

[23] L.A. Álvar-Ginés, V.M. Rafael, and E. Kjersti, "Noise robust and rotation invariant framework for texture analysis and classification," Applied Mathematics and Computation, vol. 335, pp. 124-132, 2018.

[24] A. Fakhry, T. Zeng, and S. W. Ji, "Residual deconvolutional networks for brain electron microscopy image segmentation," IEEE Trans. on Med. Imag., vol. 36, no. 2, pp. 447-456, 2017.

[25] D. Wang, T. He, Z. Li, L. Cao, N. Dey, A. S. Ashour, V. E. Balas, P. McCauley, Y. Lin, J. Xu, and F. Shi, "Image features based affective retrieval employing parameter and structure identification improved adaptive neuro fuzzy inference system," Neural Computing and Applications, vol. 29, no. 4, pp. 1087-1102, 2018.

[26] Y. Wang, Y. Chen, N. Yang, L. Zheng, N. Dey, A. S. Ashour, V. Rajinikanth, J. M. R.S. Tavares, and F. Shi, "Classification of Mice Hepatic Granuloma Microscopic Images based on Convolutional Neural Networks", Applied Soft Computing, vol.74, pp. 40-50, 2019 